\title{
INITIAL TESTS OF THE DUAL-SWEEP STREAK CAMERA SYSTEM PLANNED FOR APS PARTICLE-BEAM DIAGNOSTICS*
}

\author{
A. Lumpkin, B. Yang, and W. Gai \\ Argonne National Laboratory, 9700 S. Cass, Argonne, IL 60439 USA \\ W. Cieslik \\ Hamamatsu Photonic Systems, Bridgewater, NJ 08807 USA
}

\section{Abstract}

Initial tests of a dual-sweep streak system planned for use on the Advanced Photon Source (APS) have been performed using assets of the Argonne Wakefield Accelerator (AWA) facility. The short light pulses from the photoelectric injector drive laser in both the visible ( $\lambda=496 \mathrm{~nm}, \Delta \mathrm{t} \sim 1.5 \mathrm{ps}$ (FWHM)), and the ultraviolet $(\lambda=248 \mathrm{~nm}, \Delta t \sim 5$ ps (FWHM)) were used. Both a UV-visible S20 photocathode streak tube and a UV-to$\mathrm{x}$-ray Au photocathode streak tube were tested. Calibration data with an etalon were also obtained. A sample of dualsweep streak data using optical synchrotron radiation on the APS injector synchrotron is also presented.

\section{INTRODUCTION}

The Advanced Photon Source will be a third-generation synchrotron radiation facility for the hard $x$-ray $(10-100 \mathrm{keV})$ research community. The need to measure and monitor particle and photon beam parameters in the single bunch (10 ps), bunch-to-bunch ( 3 to $180 \mathrm{~ns}$ ), and turn-by-turn ( $3.68 \mu \mathrm{s}$ ) timescales has resulted in the choice of a dual-sweep streak camera system [1]. Initial laboratory tests with 50- and 80-ps (FWHM) laser diodes were performed. Tests at the Argonne Wakefield Accelerator [2,3] (AWA) using the short-pulsed photoelectric injector drive laser were undertaken to test both UV-visible (S20) and UV-x-ray (Au-based) photocathode streak tubes. The 1.5-ps (FWHM), 496-nm component and the 5-ps (FWHM), 248-nm component were used. Plans to use bremsstrahlung $\mathrm{X}$-rays generated by the linac beam in a short pulsed mode hitting a foil were limited by inadequate photon statistics in the first geometry tried.

\section{EXPERIMENTAL BACKGROUND}

The initial evaluations of the streak camera were with laser diodes whose bunch lengths were many times longer than the specified camera resolution. The nominal 1.5 ps (FWHM) resolution could be better evaluated with a short bunch in the 1-2 ps regime that was available at the AWA.

The AWA project in its early phase includes an L-band, 20-MeV drive linac with a high brightness photoelectric injector (PEI) capable of delivering 2-MeV, 100-nC, 20-ps (FWHM) bunches to the linac (see Fig. 1). The drive laser for this source is a pulsed laser system constructed jointly by Coherent-Lambda Physics which is described in [3]. A harmonic tripled mode-locked Nd:YAG laser is used to pump the

* Work supported by the U.S. Department of Energy, Office of Basic Energy Sciences, under Contract No. W-31-109-ENG-38. dye laser. For our test purposes, the laser was adjusted to provide 1-2 ps (FWHM) pulses at $496 \mathrm{~nm}$. Amplification of the subsequent short UV pulses at $248 \mathrm{~nm}$ was done in a single stage $\mathrm{KrF}$ excimer laser whose observed output pulse length was 4 to 5 ps (FWHM).

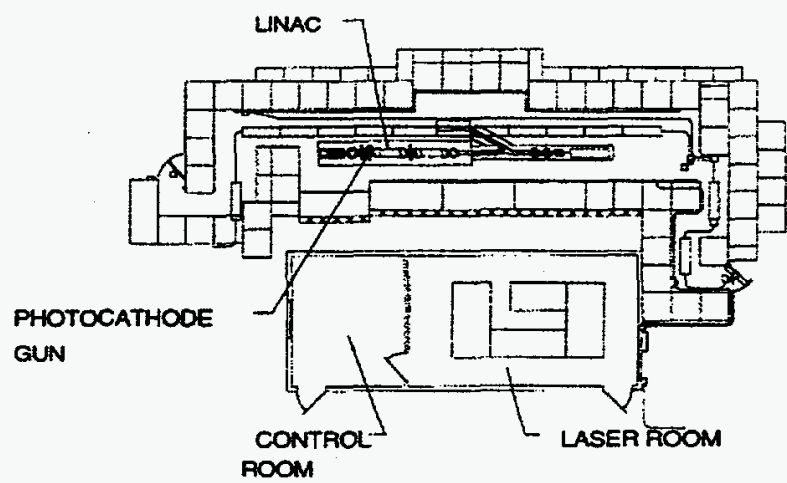

Figure 1: Schematic of the Argonne Wakefield Accelerator Facility. The drive laser and lab, the control room, and the accelerator in the shielded tunnel are indicated.

In our initial setup, as shown in Fig. 2, we used the amplified, 248-nm component from the drive laser system to evaluate the streak camera tubes' resolutions. An autocorrelator that was on-line, but sampling the green component, served as an independent bunch length monitor. The Hamamatsu C5680 with a single-shot fast sweep plug-in unit was aligned to the laser beam. A beam splitter was used to provide both a signal to a photodiode whose output generated an electrical trigger for the camera sweep and a laser beam that was appropriately delayed by transport distance to the entrance slit of the streak tube.

In a second mode, shown in Fig. 3, we split off part of the dye laser component at $496 \mathrm{~nm}$ which was also being monitored by the autocorrelator. The autocorrelator monitor nominally indicated bunch lengths of 1 to 2 ps (FWHM) in the baseline operating mode.

Both the UV-visible (S20) photocathode (PC) tube and the Au photocathode tube were evaluated. In the latter case, a quartz window on the front flange allowed UV photons to hit the PC. We also used a front flange with a Be window for the planned test with $\mathrm{x}$-rays. A portable pumping station was used to take the tube pressure to $2 \times 10^{-7}$ Torr. For both these tests, the camera was positioned off-axis near the end of the linac. Part of the drive laser beam for the PEI was directed to the streak camera.

The submitted manuscript has been authored The submited manuscipt has ben authored by a contractor of the U. Si-109 ENG-38. under contract No. W-3i-109-NG.38. Accordingly, the U.S. Government retains a nonexclusive, royalty-free license to publish or reproduce the published form of this contribution, or altow others to do 50 , for U. S. Government purposes. 


\section{DISCLAIMER}

This report was prepared as an account of work sponsored by an agency of the United States Government. Neither the United States Government nor any agency thereof, nor any of their employees, makes any warranty, express or implied, or assumes any legal liability or responsibility for the accuracy, completeness, or usefulness of any information, apparatus, product, or process disclosed, or represents that its use would not infringe privately owned rights. Reference herein to any specific commercial product, process, or service by trade name, trademark, manufacturer, or otherwise does not necessarily constitute or imply its endorsement, recommendation, or favoring by the United States Government or any agency thereof. The views and opinions of authors expressed herein do not necessarily state or reflect those of the United States Government or any agency thereof. 


\section{DISCLAIMER}

Portions of this document may be illegible in electronic image products. Images are produced from the best available original document. 


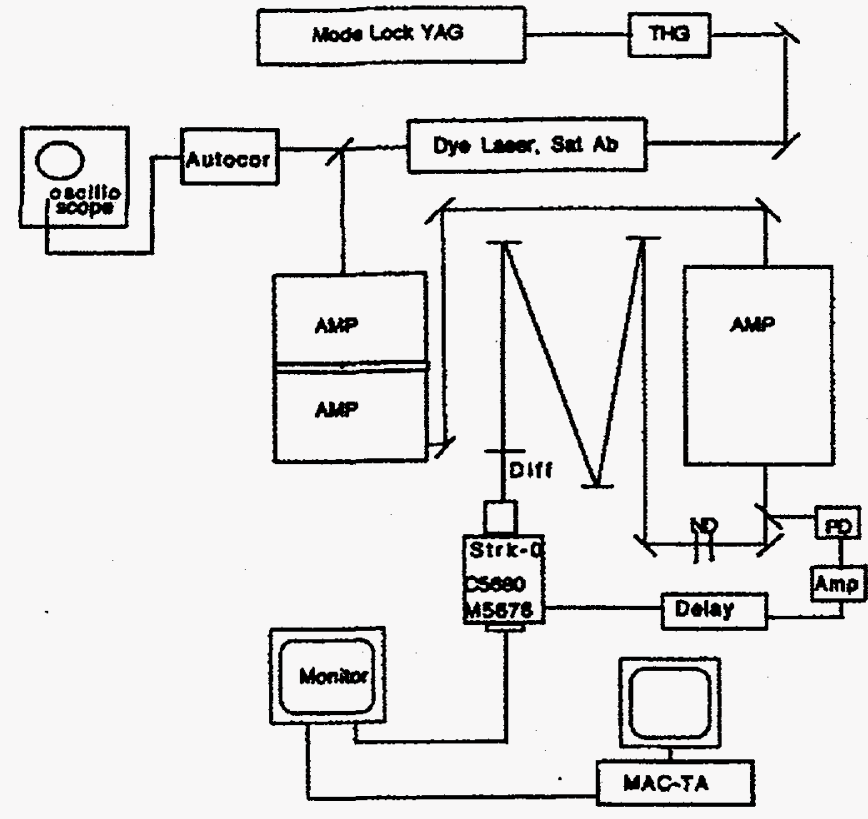

Figure 2: Initial setup for measurements on the amplified UV component $(248 \mathrm{~nm})$ of the PEI drive laser.

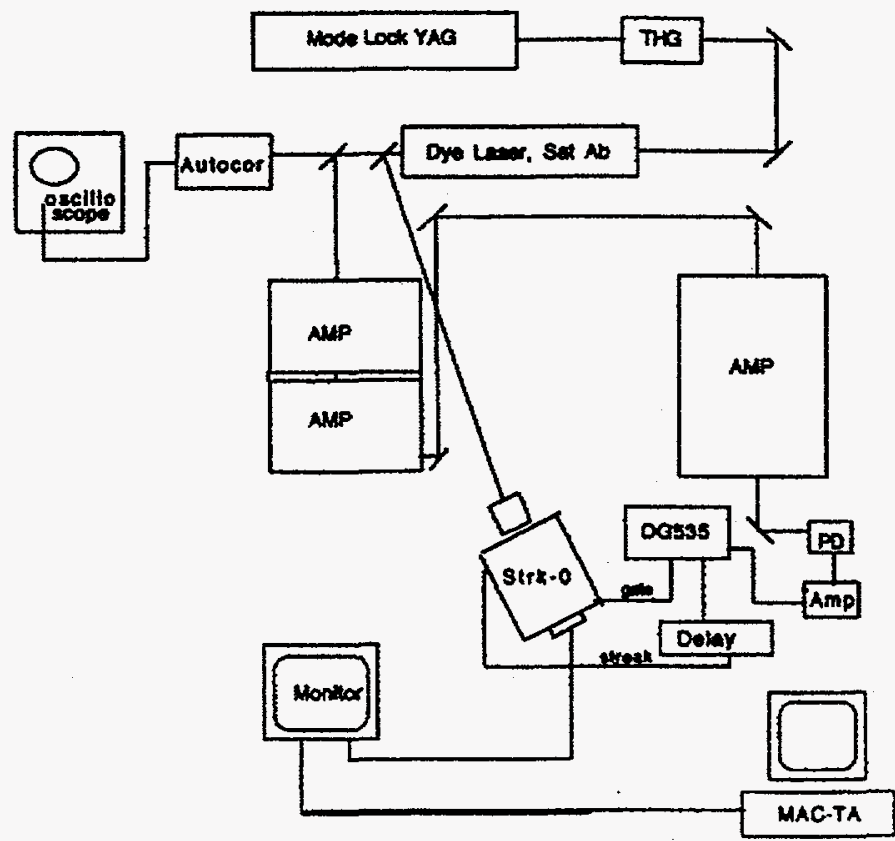

Figure 3: Setup for measurements on the 496-nm wavelength component in the drive laser.

The streak camera's information was readable by a chargecoupled device (CCD) camera, and the video digitized with a Hamamatsu MAC temporal analyzer (TA). The U5568 software program was designed for use with the Macintosh computer and the IQ-V50 frame grabber board. The system also provided remote control of most streak camera functions (a)

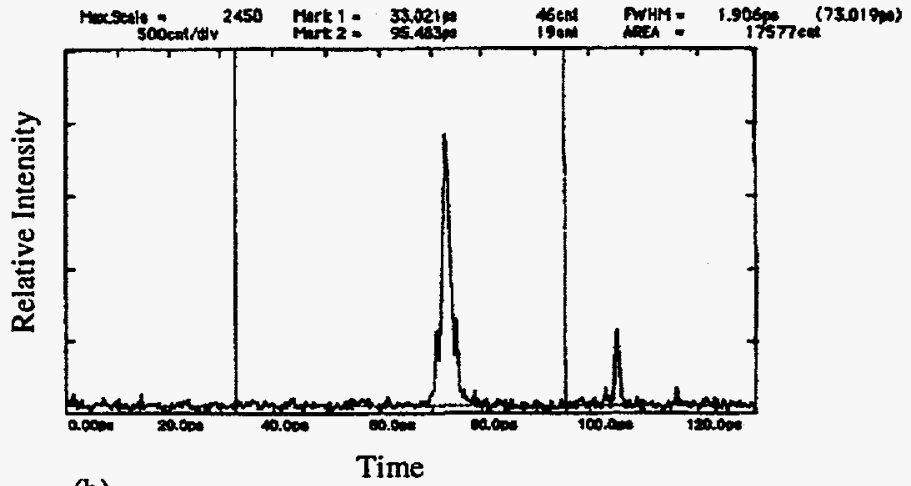

(b)



Figure 4: Profiles from streak images of the (a) green component at 1.9 ps (FWHM) and (b) example of 50-ps etalon-based calibration data. The calibration data were similar to the factory calibration since the markers centered on two peaks are 49.9 ps apart based on the calibration file.

through a GPIB interface. The image analysis program was used to provide initial evaluation of streak image position and profiles.

\section{PRELIMINARY RESULTS/DISCUSSIONS}

The initial measurements were performed on the S20 tube. In Fig. 4, a sample temporal profile from a streak image of the green component is shown. The amplified UV was observed to have a larger FWHM $(\sim 4.6 \mathrm{ps})$ bunch length than the green $(\sim 1.9 \mathrm{ps})$. The green component when monitored by the autocorrelator provided measurements of 1 to $2 \mathrm{ps}$, generally.

A second phase of experiments involved the use of an etalon with various spaces between the reflecting surfaces which results in multiple streak images spaced at known separation in time. This information was used to both validate existing calibration files and to generate new files for some plug-in units. In Fig. $4 \mathrm{~b}$, the etalon spacing was 50 ps and the reference calibration gave $49.9 \mathrm{ps}$.

In Fig. 5, test data from the Au photocathode are shown. The focus mode shows the physical extent of the active surface is $80 \mu \mathrm{m} \times 6 \mathrm{~mm}$. Due to the penetrating nature of $x$-rays, defining slits in front of the $P C$ are not a practical way to control the static spread function of the tube. The limited vertical height of this photocathode addresses this issue. The observed 
(a)

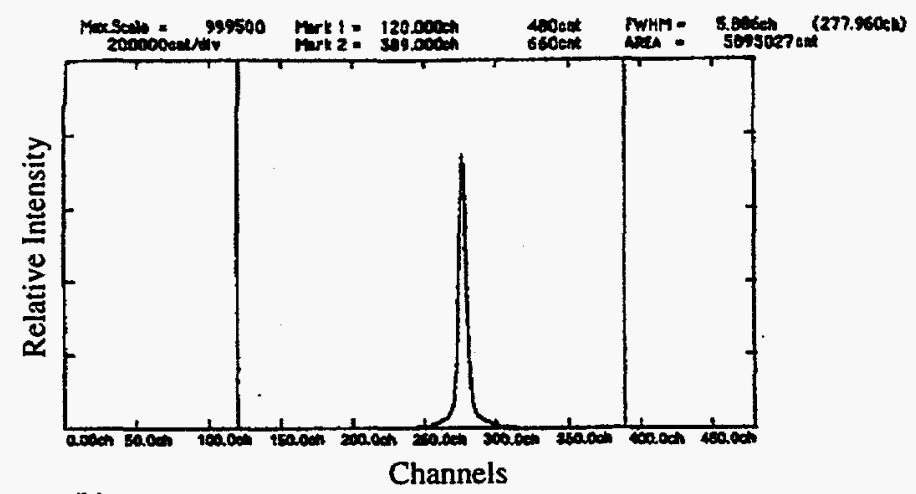

(b)

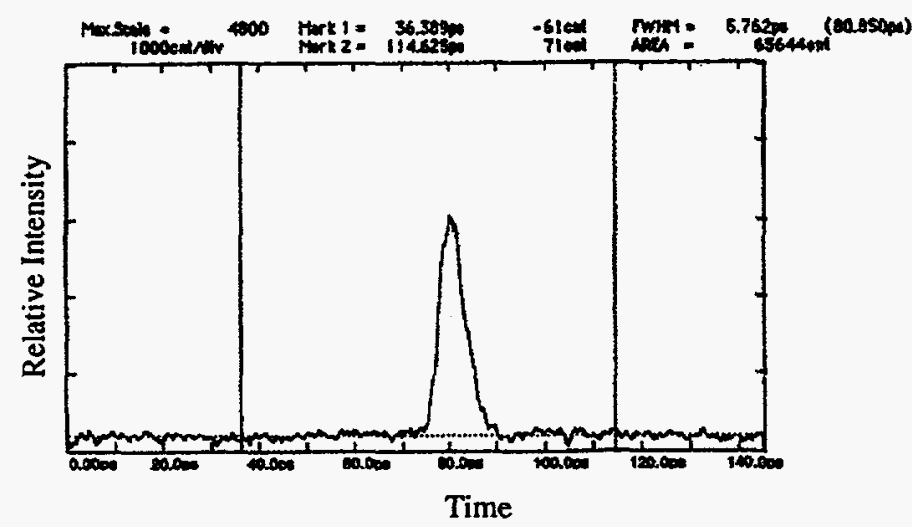

Figure 5: Temporal profiles from the Au-photocathode using the 248-nm laser irradiation: (a) focus mode with 5.9 channels FWHM and (b) streak mode with 5.8 ps (FWHM) result.

streak profile of the UV component when combined with the independent information of the S20 tube was used to determine the UV resolution to be about 2-3 ps (FWHM). In Fig. 5b, a partial laser retuning resulted in a total bunch length of $5.8 \mathrm{ps}$ (FWHM). The initial $\mathrm{x}$-ray tests using the Au PC with the Be window mounted were unsuccessful due to the limited $x$-rays that could be generated and directed to the streak camera in the available geometry.

A few months later an example of the application to APS was attained with the dual-sweep image of OSR from a bending magnet source in the injector synchrotron. The variation of bunch length during the energy ramping cycle is clearly visible and quantifiable. The damped bunch is about 158 ps (FWHM), or $\sigma=67 \mathrm{ps}$ as shown on the right-hand side of Fig. 6 . The horizontal axis spans $\sim 100 \mathrm{~ms}$ and the vertical axis $\sim 1500 \mathrm{ps}$.

\section{SUMMARY}

In summary, the AWA facility drive laser has been used to evaluate streak techniques for short-pulsed photon sources. The pulses are comparable to system resolution and faster than most baseline conditions anticipated on the APS machines. The initial evaluation on the Au photocathode with a short UV bunch was particularly useful. As a side benefit, the elongation of the amplified UV bunch length was quantified, and this will

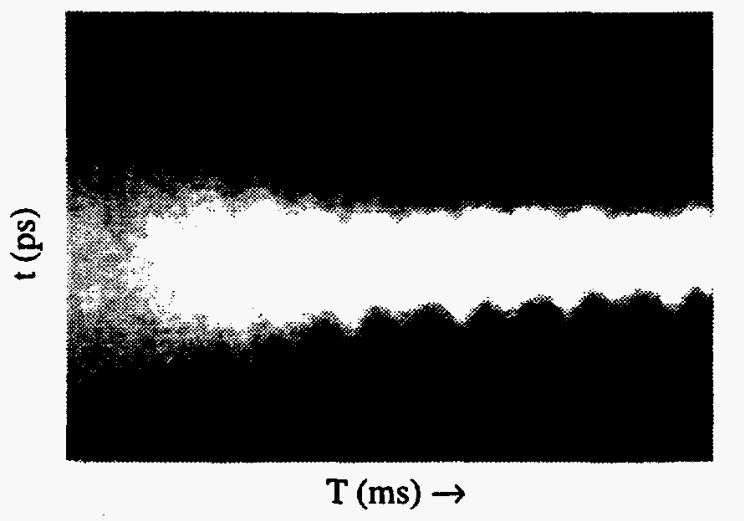

Figure 6: Dual-sweep streak image obtained of the APS injector synchrotron beam transmitting a bending magnet.

help in understanding the photoinjector performances. The first application to the APS injector synchrotron was successful in displaying bunch length dynamics, and a series of follow-on experiments on APS rings will be conducted.

\section{ACKNOWLEDGEMENTS}

The authors acknowledge Dean Walters (ASD/Vacuum Group) for providing a portable pumping station for the $\mathrm{x}$-ray tube and assisting in the vacuum issues. They also acknowledge J. Simpson for providing time on the AWA facility for these tests.

\section{REFERENCES}

[1] A.H. Lumpkin, "Characterization Techniques for the High-Brightness Particle Beam of the APS," SPIE, Vol. 2013/3, 1993.

[2] P. Schoessow, et al., "The Argonne Wakefield Accelerator-Overview and Status," Proceedings of the 1993 PAC, p. 2596, Vol. 5, 1993.

[3] W. Gai, N. Hill, C. Ho, P. Schoessow, and J. Simpson, "The AWA Laser System and Its Laser Pulse Shaper," Proceedings of the 1993 PAC, p. 3050, Vol. 4, 1993. 